\title{
Injectivity Radius of Representations of Triangle Groups and Planar Width of Regular Hypermaps
}

\author{
Martin Mačaj \\ Comenius University, Bratislava, Slovakia \\ Jozef Širáň \\ Open University, UK, and STU, Bratislava, Slovakia \\ Mária Ipolyiová \\ Matej Bel University, Banská Bystrica, Slovakia
}

Received 3 January 2008, accepted 19 December 2008, published online 31 December 2008

\begin{abstract}
We develop a rigorous algebraic background for representations of triangle groups in linear groups over algebras arising from factor rings of multivariate polynomial rings. This is then used to substantially improve the existing upper bounds on the order of epimorphic images of triangle groups with a given injectivity radius and, analogously, the size of the associated hypermaps of a given type with a given planar width.
\end{abstract}

Keywords: Triangle group, epimorphism, injectivity radius, regular hypermap, planar width.

Math. Subj. Class.: 05C10, 05C25, 05C65, 05E99, 20 C99

\section{Introduction}

The theory of regular maps and hypermaps has become increasingly important due to connections with hyperbolic geometry, group theory, Riemann surfaces, and Galois theory. These and many other links between regular (hyper)maps and other parts of mathematics are surveyed, for example, in [8]. Research in this area has, for the most part, focused on classification problems. Three most prominent (but by far not the only) examples are classification of regular embeddings of complete graphs [6], classification of regular hypermaps over 2dimensional special linear groups over finite fields [13], and classification of regular maps on surfaces with negative prime Euler characteristic [2].

E-mail addresses: martin.macaj@fmph.uniba.sk (Martin Mačaj), jozef.siran@ stuba.sk (Jozef Širáň), maria.ipolyiova@umb.sk (Mária Ipolyiová) 
In this paper we will study a phenomenon of a different nature. Informally, we will be interested in the problem of estimating the number of vertices of regular hypermaps containing 'parts' of prescribed 'size' which look 'locally planar'. We will explain the terms in quotation marks in what follows. For now we just note that there are exciting connection of this problem with finite representations of triangle groups, theory of matrices over algebraic number fields, arc-transitive graphs of given girth, and vertex-transitive non-Cayley graphs.

We begin with a description of certain hyperbolic tessellations. A triple $(l, m, n)$ of positive integers is said to be hyperbolic if $1 / l+1 / m+1 / n<1$. With any such hyperbolic triple we associate a trivalent tessellation $U(l, m, n)$ of the hyperbolic plane $\mathcal{H}$ with a proper 3 colouring of faces as follows. All faces of colour 1, 2, and 3 are congruent $2 l$-gons, $2 m$-gons, and $2 n$-gons, respectively, such that every vertex is incident to precisely one face of each colour. The underlying trivalent 1-complex of $U(l, m, n)$ is a bipartite graph, the vertices of which split into two classes according to whether the clockwise cyclic order of colours of the incident faces is $1,2,3$ or $1,3,2$.

It is well known that the group of orientation and face colour preserving automorphisms of $U(l, m, n)$ is the triangle group $T(l, m, n)$ with full presentation

$$
T(l, m, n)=\left\langle r, s, t \mid r^{m}=s^{n}=t^{l}=r s t=1\right\rangle .
$$

Let $\varphi: T(l, m, n) \rightarrow H$ be an epimorphism onto a finite group $H$ and let $N$ be the kernel of $\varphi$. We say that $\varphi$ is torsion-free if $N$ is a torsion-free group. Any such torsion-free epimorphism $\varphi: T(l, m, n) \rightarrow H$ determines the face-3-coloured quotient hypermap $\mathcal{M}_{\varphi}=$ $U(l, m, n) / N$ of type $(l, m, n)$ in the compact, orientable surface $\mathcal{S}=\mathcal{H} / N$. Orbits of the cyclic subgroups generated by $\varphi$-images of $r, s, t$ are vertices, faces, and (hyper)edges of $\mathcal{M}$. The group $H$ acts on $\mathcal{M}$ as colour and orientation preserving automorphism group. Since $H$ acts regularly on vertices of $\mathcal{M}$ that belong to the same bipartition class, the hypermap $\mathcal{M}$ is also called regular. The fact that all finite, regular hypermaps on compact, orientable surfaces arise this way is well known and we recommend [8] for details. If $l=2$, we may contract all faces coloured 2 to points and, at the same time, collapse the quadrangles coloured 1 to edges; the resulting embedded graph forms a regular map. The regular action of $H$ on vertices of one colour class in the corresponding hypermap then manifests as a regular action on arcs.

Intuitively, with an increasing index of $N$ in $T(l, m, n)$ the quotient map $\mathcal{M}$ will contain 'locally planar' parts of increasing size. To make this observation more precise, let $K$ be the 1 -skeleton of $\mathcal{M}$. Since $(l, m, n)$ is a hyperbolic triple, the supporting surface $\mathcal{S}$ of $\mathcal{M}$ has genus at least two. For any closed, non-contractible curve $\mathcal{C}$ on $\mathcal{S}$, the number of intersections $|\mathcal{C} \cap K|$ of $\mathcal{C}$ with $K$ is always non-zero, since $\mathcal{M}$ induces a cellular decomposition of $\mathcal{S}$. Following [12] we define the planar width of $\mathcal{M}$ as the minimum of $|\mathcal{C} \cap K|$, where $\mathcal{C}$ ranges over all non-contractible closed curves on $\mathcal{S}$. An interesting question is then to determine, or at least estimate, the smallest number of vertices of a finite, regular hypermap of a hyperbolic type $(l, m, n)$ with planar width larger than $d$.

As we shall see, the same idea can also be formulated in the language of group epimorphisms, without invoking quotient hypermaps. Let $T(l, m, n)$ be presented as in (1). For any non-identity element $w \in T(l, m, n)$ let $\ell(w)$ be the smallest length of a word over the alphabet $\left\{r, r^{-1}, s, s^{-1}, t, t^{-1}\right\}$ that represents $w$ in $T(l, m, n)$. The injectivity radius of an epimorphism $\varphi: T(l, m, n) \rightarrow H$ is the largest $k$ such that $\varphi(w) \neq 1$ for all non-identity $w \in T(l, m, n)$ such that $\ell(w) \leq k$. We emphasise that the injectivity radius is a function of both the epimorphism $\varphi$ as well as the generating set $\{r, s, t\}$. Now, the interesting problem 
is to estimate the smallest order of a finite group $H$ for which there exists an epimorphism $T(l, m, n) \rightarrow H$ of injectivity radius at least $\delta$.

Let us note that the two problems have solid foundations, since for every hyperbolic triple $(l, m, n)$ there exist finite, regular hypermaps of type $(l, m, n)$ with arbitrarily large planar width, as well as epimorphisms from $T(l, m, n)$ into finite groups with arbitrarily large injectivity radius. This is a consequence of residual finiteness of hyperbolic triangle groups, which follows from a general result of [9] and also from the specific approach in [10] that we will refer to later.

Although planar width was mostly considered in the context of maps, in our brief overview of the state-of-the-art we describe the results in the equivalent language of hypermaps in which $l=2$. A covering space approach to proving existence of regular hypermaps of hyperbolic type $(2, m, n)$ and arbitrary planar width was proposed in [12], furnishing no explicit upper bound on the number of vertices. Using faithful representations of triangle groups in 3-dimensional orthogonal groups over algebraic extensions of the integers, an explicit upper bound of the form $\tilde{C}(2, m, n)^{d}$ for the smallest number of vertices of a regular map of hyperbolic type $(2, m, n)$ with planar width larger than $d$ was obtained in [14]. Exploring the same method in more detail, improvements towards a smaller order of magnitude of the functions $\tilde{C}(2, m, n)$ and, in general, $\tilde{C}(l, m, n)$, were later found in [5] for regular maps and [1] for regular hypermaps. In some sense, this is the best possible type of a bound, since it follows from [11] that the number of vertices of a regular hypermap of type $(l, m, n)$ and planar width larger than $d$ is bounded below by $\tilde{c}(l, m, n)^{d}$; we note that both $\tilde{C}(l, m, n)$ and $\tilde{c}(l, m, n)$ are independent of $d$. However, the current gap between the two quantities is enormous. From [11] we know that $\sqrt{\operatorname{lmn}}<\tilde{c}(l, m, n)$, while, say, the best result of [1] only gives $\log _{2} \tilde{C}(l, m, n)<9\left(l^{2}+m^{2}+n^{2}\right)(l+m+n) l m n$. This motivates a search for better bounds on the two parameters.

In this contribution we develop methods for improvements of the upper bound. Varying the methods and their ingredients we actually prove a number of estimates on $\tilde{C}(l, m, n)$ that are asymptotically much better that the above bound; for instance, one of our estimates has the form $\log _{2} \tilde{C}(l, m, n)<64 l m n \log _{2}(4 \mu)$, where $\mu=\max \{l, m, n\}$. We will also discuss the interaction between the orders of magnitude of the new estimates. Existence of regular maps of 'small' planar width implies the existence of relatively small arc-transitive graphs with interesting additional properties, such as large girth, or non-Cayleyness; see [14]. Our results transfer into new upper bounds on the size of such graphs; we omit the details that can be obtained by direct substitution.

Our paper is organised as follows. The relationship between injectivity radius of epimorphisms of triangle groups and planar width of hypermaps is discussed in section 2 in some detail. Section 3 is the first encounter with orthogonal representations of triangle groups over polynomial rings factored by suitable ideals generated by reciprocal polynomials. The algebraic machinery for working with such representation is introduced in section 4 . The results obtained are then applied in section 5 to obtain the first large-scale improvements over the above upper bounds. Further refinements of our methods are considered in section 6, where we not only improve the previous results but also derive estimates of a slightly different kind. The last section 7 contains a discussion on planar width of regular maps.

We will arrive at our main result on planar width through a series of step-by-step improvements. Formally, it would have been possible to present a direct proof of our best bound. Nevertheless, such an approach would result in a long and technical proof and the ideas lead- 
ing to partial improvements in various directions would be overwhelmed by the large number of computational details. Our presentation leaves sufficient room to develop these ideas separately. This way the reader will be able to compare the contribution of individual ideas to the final result.

\section{Hypermaps, planar width and injectivity radius}

Consider a finite, trivalent, face-3-coloured regular hypermap $\mathcal{M}$ of a hyperbolic type $(l, m$, $n$ ) on a compact, clockwise oriented surface $\mathcal{S}$, induced by a torsion-free epimorphism $\varphi$ : $T(l, m, n) \rightarrow H$. Denoting the $\varphi$-images of $r, s$, and $t$ by $\rho, \sigma$, and $\tau$, the automorphism group $H$ of $\mathcal{M}$ has a partial presentation of the form

$$
H=\left\langle\rho, \sigma, \tau \mid \rho^{m}=\sigma^{n}=\tau^{l}=\rho \sigma \tau=\ldots=1\right\rangle .
$$

Because $\mathcal{M}$ is of hyperbolic type and so $\mathcal{S}$ has genus at least two, there will be at least one additional relator in the presentation of $H$, as indicated by the dots. Suppose that vertices of the (bipartite) 1-skeleton of $\mathcal{M}$ have been properly two-coloured, say, black and white. Fix a black vertex, say, $u$. Since $H$ acts regularly on the black vertices, for every black vertex $v$ there is a unique $\omega \in H$ such that $v=\omega(u)$. This unique $\omega$ will be called the label of $v$ and denoted $\lambda(v)$.

Let $v_{1}, v_{2}$, and $v_{3}$ be the clockwise next black vertices on the face incident with $v$ and coloured 1,2 , and 3 , respectively. We have assumed that the colours correspond to faces of length $2 l, 2 m$, and $2 n$, in that order, and hence also to the generators $\tau, \rho$, and $\sigma$, in the same order. Then, the labels of $v_{2}, v_{3}$, and $v_{1}$ are $\omega \rho, \omega \sigma$, and $\omega \tau$, as indicated in Fig. 1. Indeed, it is easy to see that, say, $\omega \rho \omega^{-1}$ is the automorphism that takes $v=\omega(u)$ onto $v_{2}$, and then $v_{2}=\omega \rho \omega^{-1}(v)=\omega \rho(u)$, showing that $\lambda\left(v_{2}\right)=\omega \rho$ as claimed; the argument for $v_{3}$ and $v_{1}$ is similar. In other terms, for any given black vertex, the label of the clockwise next black vertex in the face coloured 2,3 , and 1 is obtained by right multiplication of the label of the given vertex by the generators $\rho, \sigma$, and $\tau$ of $H$, respectively. The reader familiar with Cayley graphs and maps will immediately notice the analogy between the adjacency defined by right multiplication by the generators in the Cayley setting and the situation in our case.

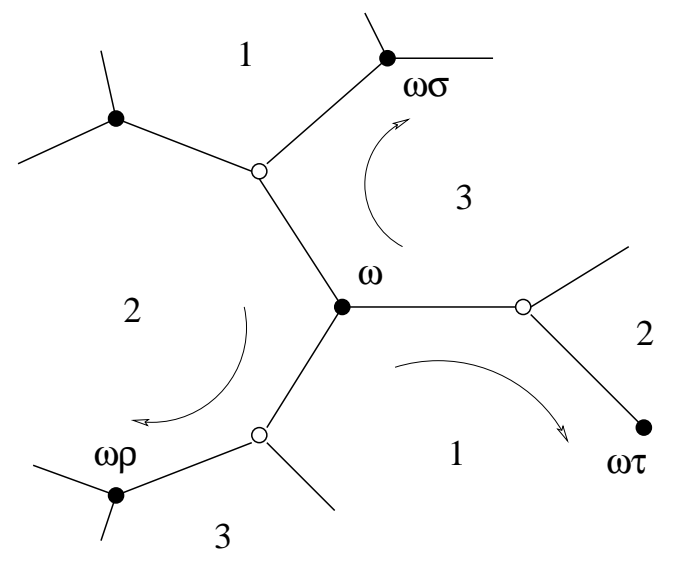

Figure 1: A fragment of a regular hypermap with group labelling of black vertices. 
The relation between elements of $H$ as words over the alphabet $\left\{\rho^{ \pm 1}, \sigma^{ \pm 1}, \tau^{ \pm 1}\right\}$ and non-contractible closed curves on the supporting surface $\mathcal{S}$ of our hypermap $\mathcal{M}$ with the 1 -skeleton $K$ can now be explained conveniently. Let $\mathcal{C}$ be a non-contractible closed curve on $\mathcal{S}$. Since we are interested in the minimum of $|\mathcal{C} \cap K|$, we may assume without loss of generality that the only points in $\mathcal{C} \cap K$ are black vertices. Let $|\mathcal{C} \cap K|=k$ and let $\mathcal{C} \cap K=\left\{v_{0}, v_{1}, \ldots, v_{k-1}\right\}$. Select a direction on $\mathcal{C}$ and choose $v_{0}$ to be the initial (and, at the same time, the terminal) point of $\mathcal{C}$. We may assume that the notation has been chosen so that $v_{i}$ and $v_{i+1}$ are consecutive for any $i(\bmod k)$ in the direction of traversal of $\mathcal{C}$. The subpath of $\mathcal{C}$ from $v_{i}$ to $v_{i+1}$ lies entirely within an open face of our hypermap; for the sake of the argument, assume that the face has colour 2 . Then, by the rule of the right multiplication by generators, we have $\lambda\left(v_{i+1}\right)=\lambda\left(v_{i}\right) \rho^{a}$ for some $a$ such that $0<|a| \leq m / 2$; the inequality on the right is strict if $m$ is odd. Repeating this successively for $i=0,1, \ldots, k-1$ for every pair of consecutive black vertices on $\mathcal{C}$ in its complete traversal from the initial point back, we arrive at the equation $\lambda\left(v_{0}\right)=\lambda\left(v_{0}\right) \gamma$ where $\gamma$ is a product of $k$ factors, each of the form $\rho^{a}$ with $0<|a| \leq m / 2$, or $\sigma^{b}$ with $0<|b| \leq n / 2$, or $\tau^{c}$ with $0<|c| \leq l / 2$. Of course, $\gamma$ is equal to the identity element of $H$, but for us the importance lies in having $\gamma$ expressed as the product of the above factors "along" the curve $\mathcal{C}$ as described. Note that a different choice of the initial point results in a conjugate of $\gamma$, and choosing the opposite direction of $\mathcal{C}$ inverts $\gamma$. This way, every such curve $\mathcal{C}$ determines a word $w$ that cannot be derived from the four relators in the partial presentation (2) for $H$. (Indeed, if $w$ was a consequence of the relators in (2), then the expression of $w$ in terms of the (conjugates of the) relators would give a way to contract $\mathcal{C}$ to a point, and vice versa.) Conversely, consider a word $w=w_{1} w_{2} \ldots w_{j}$ where $w_{i}=\alpha^{a_{i}}$ for $1 \leq i \leq j, \alpha$ is equal to $\rho, \sigma$, or $\tau$, and the $a_{i}$ are nonzero integers the absolute value of which do not exceed $l / 2, m / 2$ and $n / 2$, respectively. If $w$ represents the identity in $H$ and is independent on the four relators given in (2), then $w$ defines a non-contractible closed curve on $\mathcal{S}$ meeting $K$ at the $j$ black vertices labelled $1, w_{1}$, $w_{1} w_{2}, \ldots, w_{1} w_{2} \ldots w_{j-1}$. We sum up our observations as follows.

Lemma 1. Let $(l, m, n)$ be a hyperbolic triple, let $\mu=\max \{l, m, n\}$, and let $d$ be a positive integer. If $\varphi: T(l, m, n) \rightarrow H$ is an epimorphism onto a finite group $H$ with injectivity radius at least $d \mu / 2$, then the planar width of the hypermap $\mathcal{M}$ is larger than $d$. Conversely, if the planar width of $\mathcal{M}$ is larger than d, then the injectivity radius of $\varphi$ is at least $d$.

\section{Orthogonal representation of triangle groups}

So far we have not referred to geometric properties of universal tessellations $U(l, m, n)$ of hyperbolic type. As it is generally known [8], the triangle group $T(l, m, n)$ actually acts on $U(l, m, n)$ as the group of orientation-preserving isometries of $\mathcal{H}$, leaving the universal tessellation $U(l, m, n)$ invariant and preserving the face colours. It was shown in [10] that, in a coordinate system derived from the model of $\mathcal{H}$ as the upper-half of a two-sheeted hyperboloid $x^{2}+y^{2}-z^{2}=-1$ in $\mathcal{R}^{3}$, the generators $r, s$, and $t$ of $T(l, m, n)$ in (1) can be represented by the $3 \times 3$ matrices $R, S$, and $T$ defined by

$$
R=\left(\begin{array}{ccc}
\eta^{2}-1 & 0 & \eta \\
\zeta+\xi \eta & 1 & \xi \\
-\eta & 0 & -1
\end{array}\right), S=\left(\begin{array}{ccc}
-1 & -\zeta & 0 \\
\zeta & \zeta^{2}-1 & 0 \\
\eta & \xi+\eta \zeta & 1
\end{array}\right), T=\left(\begin{array}{ccc}
1 & \zeta & \eta+\zeta \xi \\
0 & -1 & -\xi \\
0 & \xi & \xi^{2}-1
\end{array}\right)
$$


where $\xi=2 \cos (\pi / l), \eta=2 \cos (\pi / m)$, and $\zeta=2 \cos (\pi / n)$ are algebraic integers over $\mathbb{Z}$. The group $\langle R, S, T\rangle=\langle R, S\rangle$ is a subgroup of the orthogonal group of transformations preserving the inner product defined by the quadratic form given by the matrix

$$
\left(\begin{array}{ccc}
1-\xi^{2} & \zeta+\xi \eta & \eta+\zeta \xi \\
\zeta+\xi \eta & 1-\eta^{2} & \xi+\eta \zeta \\
\eta+\zeta \xi & \xi+\eta \zeta & 1-\zeta^{2}
\end{array}\right)
$$

Combined with the general knowledge about hyperbolic geometry, it follows from [10] that the assignment $r \mapsto R, s \mapsto S$, and $t \mapsto T$ extends to a faithful representation, that is, to an injective homomorphism, from $T(l, m, n)$ to $O(3, \mathbb{R})$. This important linear representation will be invoked throughout. Two other proofs of faithfulness of this representation, both based on the much more general idea of associating canonical bilinear forms to reflection groups, can be found in [3] presenting the original approach of Tits [15], or in [4] using a more direct method.

Our goal is to construct "small" finite groups onto which there is an epimorphism from a triangle group with a given injectivity radius. The orthogonal representation introduced above seems to be an ideal candidate for this task. The main idea has roots in [9] and consists in looking at congruence subgroups, that is, normal subgroups determined by certain congruences, and at the corresponding factor groups. A way to achieve this is to regard the image of the above representation of $T(l, m, n)$ as a subgroup of $O(3, \mathbb{Z}[\xi, \eta, \zeta])$ and factorise the ring $\mathbb{Z}[\xi, \eta, \zeta]$ by an ideal generated by a suitably chosen integer.

In order to investigate injectivity radius of epimorphisms of triangle groups into finite groups arising as indicated, it turned out to be useful to adopt a more algebraic approach. Let $M=$ l.c.m. $(l, m, n)=l_{0} l=m_{0} m=n_{0} n$, let $\theta=2 \cos (\pi / M)$, and let $f_{\theta}(x) \in \mathbb{Z}[x]$ be the minimal polynomial for $\theta$. Then, $\mathbb{Z}[\xi, \eta, \zeta] \subseteq \mathbb{Z}[\theta] \cong \mathbb{Z}[x] /\left(f_{\theta}(x)\right)$, and for $\xi, \eta$ and $\zeta$ we then have $\xi=P_{l_{0}}(\theta), \eta=P_{m_{0}}(\theta)$, and $\zeta=P_{n_{0}}(\theta)$, where $P_{k}(x)$ is the $k$-th modified Tchebyshev polynomial; see [14] for details. An improvement towards smaller orders of the target groups was obtained by replacing the above ring with $\mathbb{Z}[x, y, z] / J$ where the ideal $J=(f(x), g(y), h(z))$ is chosen in such a way that, substituting $x+J, y+J$ and $z+J$ for $\xi, \eta$ and $\zeta$ in (3), the matrices arising from $R, S$ and $T$ give rise to a faithful representation of $T(l, m, n)$, cf. $[1,5]$.

To open up a way for further improvements, let us have a look at spectral properties of the matrices $R, S$, and $T$. Without loss of generality, we will confine our analysis to the matrix $S$. First, observe that the block structure of the matrix shows that $S$ is, in fact, the matrix of an affine transformation $\bar{u} \mapsto \bar{u} L+\bar{a}$ where $\bar{u} \in \mathbb{R}^{2}$ is an arbitrary two-dimensional row vector, $L$ is the submatrix of $S$ obtained by deleting the third row and the third column, and $\bar{a}=(\eta, \xi+\eta \zeta)$. Let us write $\zeta$ in the form $\zeta=\zeta_{*}+\zeta_{*}^{-1}$ where $\zeta_{*}=\cos (2 \pi / n)+i \sin (2 \pi / n)$ and $i^{2}=-1$. An easy calculation shows that the eigenvalues of $L$ are $\zeta_{*}^{2}$ and $\zeta_{*}^{-2}$ and hence the spectrum of $S$ is $\left\{1, \zeta_{*}^{2}, \zeta_{*}^{-2}\right\}$; in particular, the order of $L$ is equal to the order of $S$.

The observation about the spectrum is the cornerstone of this work, the essence of which is fusing the outlined algebraic methods with representing $\xi, \eta$ and $\zeta$ as a sum of an element and its inverse. Although our results will be valid in a more general setting, we will confine ourselves mostly to considerations in the rings $\mathbb{Z}[x] /\left(\Phi_{2 M}(x)\right)$ where $M=$ l.c.m. $(l, m, n)$ and $\Phi_{2 M}(x)$ is the $2 M$-th cyclotomic polynomial, and in $\mathbb{Z}[x, y, z] /(f(x), g(y), h(z))$ where $f(x)=\left(x^{2 l}-1\right) /\left(x^{2}-1\right), g(y)=\left(y^{2 m}-1\right) /\left(y^{2}-1\right)$ and $h(z)=\left(z^{2 n}-1\right) /\left(z^{2}-1\right)$.

The reason for choosing the two rings will become clear soon; at this stage we just show that they lead to faithful representations of type (3). From now on, we will assume that $R$, 
$S$, and $T$ are matrices as in (3), but the parameters $\xi, \eta$ and $\zeta$ appearing there will belong to more general structures (rings or fields) that will be specified in the course of our exposition. We derive simple sufficient conditions for the matrices $R, S$, and $T$ to still generate a faithful representation of $T(l, m, n)$. For convenience, we will sometimes identify the (faithful) representations of $T(l, m, n)$ with the corresponding images $\langle R, S, T\rangle$. We begin with an auxiliary result about orders of such matrices and without loss of generality we focus on $S$.

Lemma 2. Let $n \geq 2$ be an integer, let $F$ be a field of characteristic not dividing $n$, and let $S$ be the matrix in (3) such that $\xi, \eta, \zeta \in F$. Let $\zeta=\zeta_{*}+\zeta_{*}^{-1}$ where $\zeta_{*}$ is either an element of $F$ or an element of an extension of $F$ of degree two. Then, the order of $S$ divides $n$ if and only if $\zeta_{*}^{2 n}=1$ and $\zeta_{*}^{2} \neq 1$. If the characteristic of $F$ divides $n$, then the condition $\zeta_{*}^{2} \neq 1$ is no longer necessary.

Proof. Our earlier observations about the orders extend to matrices over any field. Specifically, the order of $S$ is equal to the order of the submatrix $L$ of $S$ formed by deleting the third row and the third column. The eigenvalues of $L$ are $\zeta_{*}^{2}$ and $\zeta_{*}^{-2}$. It can be checked that $L$ is conjugate to $\left(\begin{array}{ll}1 & 1 \\ 0 & 1\end{array}\right)$ if and only if $\zeta_{*}^{2}=1$, in which case the order of $L$ is equal to the characteristic of $F$. If $\zeta_{*}^{2} \neq 1$, then $L$ is conjugate to the matrix $\operatorname{Diag}\left(\zeta_{*}^{2}, \zeta_{*}^{-2}\right)$, making the conclusion about the order of $S$ obvious.

Now we are ready to state and prove the indicated results on representations of triangle groups.

Proposition 3. Let $(l, m, n)$ be a hyperbolic triple and let $M=$ l.c.m. $(l, m, n)=l_{0} l=$ $m_{0} m=n_{0} n$. Let $f(x) \in \mathbb{Z}[x]$ be a monic reciprocal polynomial of even degree. Let $R, S$, and $T$ be matrices as in (3) defined over the ring $\mathbb{Q}[x] /(f(x))$, where $\xi=x^{l_{0}}+x^{-l_{0}}+(f(x))$, $\eta=x^{m_{0}}+x^{-m_{0}}+(f(x))$, and $\zeta=x^{n_{0}}+x^{-n_{0}}+(f(x))$. If the $2 M$-th cyclotomic polynomial divides $f(x)$ and if $f(x)$ divides $\left(x^{2 M}-1\right) /$ l.c.m. $\left(x^{2 l_{0}}-1, x^{2 m_{0}}-1, x^{2 n_{0}}-1\right)$, then the assignment $r \mapsto R, s \mapsto S$ and $t \mapsto T$ extends to a faithful representation of the triangle group $T(l, m, n)$ in $S L(3, \mathbb{Q}[x] /(f(x)))$.

Proof. Let us denote by $G_{x}$ the group generated by $R, S$, and $T$. For any root $b$ of $f(x)$ let $G_{b}$ denote the group generated by the three matrices obtained from $R, S$, and $T$ by replacing $\xi, \eta$, and $\zeta$ with $b^{l_{0}}+b^{-l_{0}}, b^{m_{0}}+b^{-m_{0}}$, and $b^{n_{0}}+b^{-n_{0}}$, respectively. By the substitution theorem we have an obvious epimorphism $G_{x} \rightarrow G_{b}$. Since $f(x)$ does not have repeated roots, there is a natural embedding $G_{x} \hookrightarrow \prod G_{b}$ where the product ranges over conjugacy classes of roots of $f(x)$. Therefore, $G_{x}$ is a faithful representation of $T(l, m, n)$ if every $G_{b}$ is a representation of $T(l, m, n)$ and at least one of them is faithful. Faithfulness of at least one of $G_{b}$ follows from the condition $\Phi_{2 M}(x) \mid f(x)$, that is, from Mennicke's representation [10]. Since $f(x)$ divides $\left(x^{2 M}-1\right) /$ l.c.m. $\left(x^{2 l_{0}}-1, x^{2 m_{0}}-1, x^{2 n_{0}}-1\right)$, Lemma 2 guarantees that every $G_{b}$ is a representation of $T(l, m, n)$.

In a similar way one can prove the following result; we leave the details to the reader.

Proposition 4. Let $(l, m, n)$ be a hyperbolic triple. Let $J=(f(x), g(y), h(z))$ be an ideal in $\mathbb{Q}[x, y, z]$ where $f(x) \in \mathbb{Z}[x], g(y) \in \mathbb{Z}[y]$, and $h(z) \in \mathbb{Z}[z]$ are monic reciprocal polynomials of even degree. Let $R, S$, and $T$ be matrices as in (3) defined over the ring $\mathbb{Q}[x, y, z] / J$, where $\xi=x+x^{-1}+J, \eta=y+y^{-1}+J$, and $\zeta=z+z^{-1}+J$. Assume that $\Phi_{2 l}(x) \mid f(x)$ 
and $f(x)\left|\left(x^{2 l}-1\right) /\left(x^{2}-1\right), \Phi_{2 m}(y)\right| g(y)$ and $g(y) \mid\left(y^{2 m}-1\right) /\left(y^{2}-1\right)$, and $\Phi_{2 n}(z) \mid h(z)$ and $h(z) \mid\left(z^{2 n}-1\right) /\left(z^{2}-1\right)$. Then, the assignment $r \mapsto R, s \mapsto S$ and $t \mapsto T$ extends to $a$ faithful representation of the triangle group $T(l, m, n)$ in $S L(3, \mathbb{Q}[x, y, z] / J)$.

\section{Injectivity radius and norms in algebras}

From the methods and results of [11] used to investigate the growth rate of maps, the following fact can be extracted.

Proposition 5. Let $(l, m, n)$ be a hyperbolic triple and let $\delta$ be a positive integer. Then, for every epimorphism $\varphi: T(l, m, n) \rightarrow H$ of injectivity radius at least $\delta$ onto a finite group $H$ we have $|H|>c^{\delta}$ where $c$ depends on $l, m, n$ but not on $\delta$.

The natural question of constructing groups $H$ as above to which a similar upper bound applies was answered in the affirmative in [14] and improvements of the construction were found later in $[1,5]$. We briefly sum up the essence of the approach in the cited papers, using a more general framework.

Let $A$ be a $u$-dimensional abelian $\mathbb{Z}$-algebra, that is, an abelian ring with unity such that the additive group of $A$ is a $u$-dimensional free $\mathbb{Z}$-module and multiplication is bilinear. Let $\beta_{1}, \ldots, \beta_{u}$ be an (additive) basis of $A$. Then, every element $\alpha \in A$ can be uniquely expressed in the form $\alpha=a_{1} \beta_{1}+\ldots+a_{u} \beta_{u}$, where $a_{i} \in \mathbb{Z}$ for $1 \leq i \leq u$. The integral norm of $\alpha$ with respect to the basis $\beta_{1}, \ldots, \beta_{u}$ is the integer

$$
\langle\alpha\rangle=\max _{i}\left|a_{i}\right| .
$$

This integral norm extends in a natural way to vectors and matrices over $A$ (by simply taking the maximum norm over all entries of a vector or a matrix) and we will reserve the same notation for such extensions.

The previous work on injectivity radius $[1,5,14]$ was based on variations of the following general result.

Theorem 6. Let $A$ be a $u$-dimensional abelian $\mathbb{Z}$-algebra. Let $\vartheta: T(l, m, n) \rightarrow \operatorname{SL}(q, A)$ be a faithful representation of the triangle group $T(l, m, n)=\langle r, s, t| r^{m}=s^{n}=t^{l}=r$ st $\left.=1\right\rangle$ and let $\Gamma=\left\{\vartheta(r)^{ \pm 1}, \vartheta(s)^{ \pm 1}, \vartheta(t)^{ \pm 1}\right\}$. Further, let $\lambda=\max _{X \in \Gamma}\langle X\rangle$ where the norm is with respect to a fixed basis of $A$ containing 1 and let $\kappa=\max \left\{\langle X \alpha\rangle /\langle\alpha\rangle ; \alpha \in A^{q} \backslash\{0\}, X \in\right.$ $\Gamma\}$. Assume that there is a positive integer $\delta$ and a prime $p$ such that $\kappa^{\delta-1} \lambda+1<p<\kappa^{\delta}$. If $\psi_{p}$ is the natural projection $\mathrm{SL}(q, A) \rightarrow \mathrm{SL}(q, A / p A)$, then the representation $\psi_{p} \vartheta$ has injectivity radius at least $\delta$ and $\left|\operatorname{Im}\left(\psi_{p} \vartheta\right)\right|<C^{\delta}$ where $C=\kappa^{\left(q^{2}-1\right) u}$.

Proof. It is an easy exercise to show that, for an integral norm on $A$ with respect to an arbitrary basis, the parameter $\kappa$ is well defined and $\kappa \geq \lambda \geq 1$. To prove the theorem, it is sufficient to show that for any $j \leq \delta$ and any $X_{1}, X_{2}, \ldots, X_{j} \in \Gamma$ such that $X_{j} \ldots X_{2} X_{1} \neq I$ in $\operatorname{SL}(q, A)$ we have $\left\langle X_{j} \ldots X_{2} X_{1}-I\right\rangle<p$; this will guarantee that the product modulo $p A$ cannot be equal to the identity matrix. Let $\alpha$ be any column of $X_{1}$. By induction we obtain $\left\langle X_{j} \ldots X_{2} \alpha\right\rangle \leq \kappa^{j-1}\langle\alpha\rangle \leq \kappa^{\delta-1} \lambda$, which implies that $\left\langle X_{j} \ldots X_{2} X_{1}\right\rangle \leq \kappa^{\delta-1} \lambda$. If $p$ is a prime such that $\kappa^{\delta-1} \lambda+1<p<\kappa^{\delta}$, then all integral coefficients in all entries of the matrix $X_{j} \ldots X_{2} X_{1}-I$ are, in absolute value, smaller than $p$. Since the basis of $A$ is assumed to contain 1 , no nonzero integral coefficient in any entry of the above matrix mod $p A$ collapses. Therefore $X_{j} \ldots X_{2} X_{1} \neq I$ in $\operatorname{SL}(q, A / p A)$; for the order of this group we 
have $|\operatorname{SL}(q, A / p A)| \leq|A / p A|^{q^{2}-1}=p^{\left(q^{2}-1\right) u}<\kappa^{\left(q^{2}-1\right) u \delta}$.

In section 3 we indicated that earlier improvements on the bounds of injectivity radius of triangle groups given in [14] focused on factor rings associated with minimal polynomials of algebraic integers of type $2 \cos \pi / k$. This way the best construction in [1] gives, after a rectification in the theoretical part of the argument, a finite group $H$ admitting an epimorphism $\varphi: T(l, m, n) \rightarrow H$ of injectivity radius at least $\delta$ such that

$$
|H|<C^{\delta} \text { where } \log _{2} C<9\left(l^{2}+m^{2}+n^{2}\right) l m n .
$$

Restricting to $l=2$ and denoting by $\phi$ the Euler's function, the analogous best result of [5] yields

$$
|H|<C^{\delta} \quad \text { where } \quad \log _{2} C<2\left(\phi(2 m)^{2}+\phi(2 n)^{2}\right) \phi(2 m) \phi(2 n) .
$$

Since the original bound in [14] for $l=2$ was $\log _{2}(C)<72(m n)^{3}$, the contribution of (5) consists, roughly speaking, in replacing the factor $(m n)^{2}$ by $(m+n)^{2}$. The actual improvement therefore depends on the order of magnitude of the ratio between $m$ and $n$.

With the help of the two faithful representations of hyperbolic triangle groups introduced in section 3, in the next section we will derive a substantial improvement of these constructions. We will do so by applying Theorem 6 to the $\mathbb{Z}$-algebras used in Propositions 3 and 4. For this we need to prove estimates on integral norms with respect to suitable bases. In view of the general philosophy outlined in Section 3 it should be no surprise that the bases will be formed by sums of certain elements and their inverses.

Consider a monic, reciprocal polynomial $f(x) \in \mathbb{Z}[x]$ of even degree $2 u$, given by

$$
f(x)=x^{2 u}+c_{1} x^{2 u-1}+\cdots+c_{u-1} x^{u+1}+c_{u} x^{u}+c_{u-1} x^{u-1}+\cdots+c_{1} x+1 .
$$

Reciprocality allows us to work with elements of the form $x+x^{-1}+(f(x))$ in the ring $\mathbb{Z}[x] /(f(x))$. Let

$$
\theta=x+x^{-1}+(f(x)) \text { and } \theta_{i}=x^{i}+x^{-i}+(f(x))
$$

for any $i \in \mathbb{Z}$; in particular, $\theta_{0}=2$ and $\theta_{1}=\theta$. The set $\mathcal{B}=\left\{1, \theta_{1}, \theta_{2}, \ldots, \theta_{u-1}\right\}$ forms a basis of the $\mathbb{Z}$-algebra $\mathbb{Z}[\theta]$. The following obvious relations between the elements of $\mathcal{B}$ will help us simplify expressions involving multiplication:

$$
\begin{aligned}
\theta_{i} \theta_{j} & =\theta_{i+j}+\theta_{i-j}, \\
\theta_{u} & =-c_{u}-\sum_{i=1}^{u-1} c_{u-i} \theta_{i}, \\
\theta_{i} & =\theta_{-i}, \\
\theta_{i+1} & =\theta_{1} \theta_{i}-\theta_{i-1} .
\end{aligned}
$$

The norm \langle\rangle we will be considering below will always be relative to the basis $\mathcal{B}$. To simplify the notation, we will write $\langle f\rangle$ instead of $\langle f(x)\rangle=\max _{i}\left|c_{i}\right|$, and $\langle g\rangle$ instead of $\langle g(\theta)\rangle$ for elements $g(\theta) \in \mathbb{Z}[\theta]$. 
Lemma 7. Let $i$ be a positive integer and let $g=g(\theta)=a_{0}+\sum_{j=1}^{u-1} a_{j} \theta_{j}$ where $a_{0}$ and the $a_{j}$ are integers. Then

$$
\begin{aligned}
\left\langle\theta_{1} g(\theta)\right\rangle & \leq(2+\langle f\rangle)\langle g\rangle \\
\left\langle\theta_{u+i}\right\rangle & \leq(3+\langle f\rangle)^{i}\langle f\rangle \\
\left\langle\theta_{i} g(\theta)\right\rangle & \leq\left(2+\langle f\rangle \frac{(3+\langle f\rangle)^{i}-1}{2+\langle f\rangle}\right)\langle g\rangle<(3+\langle f\rangle)^{i}\langle g\rangle .
\end{aligned}
$$

Proof. With the help of (6) and (8) we obtain

$$
\theta_{1} g(\theta)=2 a_{1}+\sum_{i=1}^{u-2}\left(a_{i-1}+a_{i+1}\right) \theta_{i}+a_{u-2} \theta_{u-1}+a_{u-1} \theta_{u}
$$

and applying (7) yields (10). To prove (11) it suffices to repeatedly apply (9) and (10), starting with $\left\langle\theta_{u}\right\rangle=\langle f\rangle$. For the last inequality, using (6) and (8) we arrive at

$$
\left\langle\theta_{i} g(\theta)\right\rangle \leq 2\langle g\rangle+\left(\left\langle\theta_{u}\right\rangle+\cdots+\left\langle\theta_{u+i-1}\right\rangle\right)\langle g\rangle .
$$

Combined with (11), the above transforms into

$$
\left\langle\theta_{i} g(\theta)\right\rangle \leq\left(2+\langle f\rangle \frac{(3+\langle f\rangle)^{i}-1}{2+\langle f\rangle}\right)\langle g\rangle,
$$

which is the first part of (12). The last inequality in (12) is obvious.

We conclude with another note regarding the earlier work on bounding the injectivity radius. In $[1,5,14]$, modifications of Theorem 6 were used relative to the basis $\left\{1, \theta, \theta^{2}, \ldots\right.$, $\left.\theta^{u-1}\right\}$. In contrast with this, here we will be using the basis $\left\{1, \theta_{1}, \theta_{2}, \ldots, \theta_{u-1}\right\}$. As we shall see, and as one may have anticipated from the outlined relation of the new basis with spectra, the effect of the change of basis will be considerable.

\section{New bounds on injectivity radius}

We are now prepared to improve the bounds (4) on the injectivity radius and the associated target group. Our point of departure will be Propositions 3 and 4 where we introduced two representations of triangle groups; from now on we will call them accordingly Representation 1 and 2.

We begin with Representation 1. Let $(l, m, n)$ be a hyperbolic triple and let $M=$ l.c.m. $(l, m, n)=l_{0} l=m_{0} m=n_{0} n$. Let $f(x)=\Phi_{2 M}(x) \in \mathbb{Z}[x]$ be the $2 M$-th cyclotomic polynomial of degree $\phi(2 M)=2 u$. As in the previous section, we define $\theta=x+x^{-1}+(f(x))$ and $\theta_{i}=x^{i}+x^{-i}+(f(x))$ for any $i \in \mathbb{Z}$ and consider the basis $\mathcal{B}=\left\{1, \theta_{1}, \theta_{2}, \ldots, \theta_{u-1}\right\}$ of the $\mathbb{Z}$-algebra $\mathbb{Z}[\theta]$ together with the associated integral norm \langle\rangle . Further, let $R, S$, and $T$ be matrices as in (3) over $\mathbb{Z}[\theta]$, where $\xi=\theta_{l_{0}}, \eta=\theta_{m_{0}}$, and $\zeta=\theta_{n_{0}}$. At last, let $\mu_{0}=\max \left\{l_{0}, m_{0}, n_{0}\right\}$. In what follows we derive estimates on the parameters $\kappa, \lambda$, and $C$ introduced in Theorem 6 .

We first show that $\kappa \leq(3+\langle f\rangle)^{2 \mu_{0}}$. To this end, it is sufficient to show that $\langle X \alpha\rangle<$ $(3+\langle f\rangle)^{2 \mu_{0}}\langle\alpha\rangle$ for every $X \in\left\{R^{ \pm 1}, S^{ \pm 1}, T^{ \pm 1}\right\}$ and every 3-dimensional column vector $\alpha$ with entries $g_{j}=g_{j}(\theta) \in \mathbb{Z}[\theta]$ for $j=1,2,3$. We outline the calculations for the matrix 
$X=S$ only, as the particulars for the remaining five matrices are analogous. To simplify the matters, in estimating $\langle S \alpha\rangle$ we will restrict ourselves to the contribution coming from the third row of $S$. Thus, we will replace $\langle S \alpha\rangle$ with the scalar product $\left\langle S_{(3)} \alpha\right\rangle$ where $S_{(3)}$ is the third row of $S$; from our computations it will be obvious that the resulting upper bound on $\left\langle S_{(3)} \alpha\right\rangle$ will also be an upper bound on $\langle S \alpha\rangle$. Also, if $g \in\left\{g_{1}, g_{2}, g_{3}\right\}$ is such that $\langle\alpha\rangle=\langle g\rangle$, then in our estimates we may for simplification assume that all entries of $\alpha$ are equal to $g$. We then have

$$
\left\langle S_{(3)} \alpha\right\rangle \leq\langle(\eta+(\xi+\eta \zeta)+1) g(\theta)\rangle \leq\langle g\rangle+\langle\eta g(\theta)\rangle+\langle\xi g(\theta)\rangle+\langle\eta \zeta g(\theta)\rangle .
$$

For any positive integer $i$ let $\left.\chi_{i}=2+(3+\langle f\rangle)^{i}-1\right)\langle f\rangle /(2+\langle f\rangle)$. By (12), we have $\langle\eta g(\theta)\rangle=\left\langle\theta_{m_{0}} g(\theta)\right\rangle \leq \chi_{m_{0}}\langle g\rangle,\langle\xi g(\theta)\rangle=\left\langle\theta_{l_{0}} g(\theta)\right\rangle \leq \chi_{l_{0}}\langle g\rangle$, and $\langle\eta \zeta g(\theta)\rangle=$ $\left\langle\theta_{m_{0}} \theta_{n_{0}} g(\theta)\right\rangle \leq \chi_{m_{0}} \chi_{n_{0}}\langle g\rangle$. This yields

$$
\left\langle S_{(3)} \alpha\right\rangle \leq\left(1+\chi_{l_{0}}+\chi_{m_{0}}+\chi_{m_{0}} \chi_{n_{0}}\right)\langle\alpha\rangle .
$$

A routine calculation shows that $1+\chi_{l_{0}}+\chi_{m_{0}}+\chi_{m_{0}} \chi_{n_{0}} \leq(3+\langle f\rangle)^{2 \mu_{0}}$, and hence $\left\langle S_{(3)} \alpha\right\rangle \leq(3+\langle f\rangle)^{2 \mu_{0}}\langle\alpha\rangle$. Similarly, $\left\langle S_{(1)} \alpha\right\rangle \leq\left(1+\chi_{n_{0}}\right)\langle\alpha\rangle \leq(3+\langle f\rangle)^{2 \mu_{0}}$ and $\left\langle S_{(2)} \alpha\right\rangle \leq\left(1+\chi_{n_{0}}+\chi_{2 n_{0}}\right)\langle\alpha\rangle \leq(3+\langle f\rangle)^{2 \mu_{0}}$. It is now clear that the right hand side of the last inequality is an upper bound on $\langle S \alpha\rangle$ as well, which proves that $\kappa \leq(3+\langle f\rangle)^{2 \mu_{0}}$.

We continue our analysis by estimating $\lambda=\max \langle X\rangle$ for $X \in\left\{R^{ \pm 1}, S^{ \pm 1}, T^{ \pm 1}\right\}$. If $2 \mu_{0}<u=\phi(2 M) / 2$, then applying (6) to entries of $X$ we obtain $\lambda \leq 2$. If $2 \mu_{0} \geq u$, then the inequality (11) of Lemma 7 in combination with the observation that $u \geq 2$ for all hyperbolic triples shows that $\lambda \leq 3+(3+\langle f\rangle)^{\mu_{0}-u}\langle f\rangle+(3+\langle f\rangle)^{2 \mu_{0}-u}\langle f\rangle \leq(3+\langle f\rangle)^{2 \mu_{0}-1}$. Summing up our discussion, we conclude that $\lambda \leq(3+\langle f\rangle)^{2 \mu_{0}-1}$ for all hyperbolic triples. Set $\kappa_{0}=(3+\langle f\rangle)^{2 \mu_{0}}$ and $\lambda_{0}=(3+\langle f\rangle)^{2 \mu_{0}-1}$. By Bertrand's Postulate, for any positive integer $\delta \geq 1$ there exists a prime $p$ between $\kappa_{0}^{\delta-1} \lambda_{0}+1$ and $\kappa_{0}^{\delta}$. We may, of course, apply Theorem 6 with the upper bounds $\kappa_{0}$ and $\lambda_{0}$ in place of $\kappa$ and $\lambda$, arriving thus at our first specific result.

Theorem 8. Let $(l, m, n)$ be a hyperbolic triple, let $M=1 . c . m .(l, m, n)=l_{0} l=m_{0} m=$ $n_{0} n$, and let $\mu_{0}=\max \left\{l_{0}, m_{0}, n_{0}\right\}$. Let $f(x)$ be the $2 M$-th cyclotomic polynomial and let $\langle f\rangle$ be the largest absolute value of its coefficients. Then, for every positive integer $\delta$ there exists an epimorphism $\varphi: T(l, m, n) \rightarrow H$ of injectivity radius at least $\delta$ such that $|H| \leq C^{\delta}$ where $C \leq(3+\langle f\rangle)^{8 \mu_{0} \phi(2 M)}$.

The corresponding result for the existence of relatively "small" regular hypermaps of planar width larger than a preassigned integer follows immediately from the preceding theorem and Lemma 1.

Corollary 9. Let $(l, m, n)$ be a hyperbolic triple, let $\mu=\max \{l, m, n\}$, let $M=$ l.c.m. $(l, m$, $n)=l_{0} l=m_{0} m=n_{0} n$, and let $\mu_{0}=\max \left\{l_{0}, m_{0}, n_{0}\right\}$. Let $f(x)$ be the $2 M$-th cyclotomic polynomial and let $\langle f\rangle$ be the largest absolute value of its coefficients. Then, for every positive integer $d$ there exists a regular hypermap of type $(l, m, n)$ of planar width larger than $d$, the number of vertices of which does not exceed $2 \tilde{C}^{d}$ where $\tilde{C} \leq(3+\langle f\rangle)^{4 \mu \mu_{0} \phi(2 M)}$.

The estimates in Theorem 8 and Corollary 9 clearly supersede the improvements (4) and (5) of the original bound given in [14]. The exact order of magnitude of the bounds on $C$ is, however, not easy to extract without a more detailed information about coefficients in 
cyclotomic polynomials, and we will address this issue later. Nevertheless, this provides motivation for finding good bounds the order of magnitude of which would be more obvious. We do so by focusing on Representation 2.

Again, let $(l, m, n)$ be a hyperbolic triple. Let $f(x) \in \mathbb{Z}[x], g(y) \in \mathbb{Z}[y]$, and $h(z) \in$ $\mathbb{Z}[z]$ be defined by $f(x)=\left(x^{2 l}-1\right) /\left(x^{2}-1\right), g(y)=\left(y^{2 m}-1\right) /\left(y^{2}-1\right), h(z)=$ $\left(z^{2 n}-1\right) /\left(z^{2}-1\right)$. All the three polynomials are monic, reciprocal, and of even degree; let $u_{f}=\operatorname{deg}(f) / 2, u_{g}=\operatorname{deg}(g) / 2, u_{f}=\operatorname{deg}(h) / 2$, and $u=u_{f} u_{g} u_{h}<l m n$. Consider the ideal $J=(f(x), g(y), h(z)) \in \mathbb{Q}[x, y, z]$ and let $\xi=x+x^{-1}+J, \eta=y+y^{-1}+J$, and $\zeta=z+z^{-1}+J$. Similarly, for any integers $i, j, k$ we define $\xi_{i}=x^{i}+x^{-i}+J$, $\eta_{j}=y^{j}+y^{-j}+J$, and $\zeta_{k}=z^{k}+z^{-k}+J$. We will now be working with the $\mathbb{Z}$-algebra $A=\mathbb{Z}[\xi, \eta, \zeta]$. The set $\mathcal{B}$ of all products $\xi_{i} \eta_{j} \zeta_{k} / 2^{\varepsilon}$ where $0 \leq i<u_{f}, 0 \leq j<u_{g}$, $0 \leq k<u_{h}$, with $\varepsilon$ equal to the number of subscripts $i, j, k$ in $\xi_{i} \eta_{j} \zeta_{k}$ equal to 0 , forms a basis of $A$ containing 1; the dimension of $A$ is equal to $u$.

Results of Lemma 7 carry over to bounds on the norm of elements of $A=\mathbb{Z}[\xi, \eta, \zeta]$ with a minor modification of the argument. We will therefore invoke it in our subsequent considerations regarding Representation 2.

By (6) we have $\xi^{2}-1=\xi_{2}+1, \eta^{2}-1=\eta_{2}+1$, and $\zeta^{2}-1=\zeta_{2}+1$. If $l \geq 4$, then $\xi_{2} \in \mathcal{B}$. If $l=2$ or 3 , a calculation shows that $\xi_{2}+1=1$ or 0 , respectively. The similar holds for $m$ and $n$, and therefore $\lambda=1$ for any hyperbolic triple.

An application of the inequalities (10) and (12) of Lemma 7 shows that for every $g \in A$ and for every $\varepsilon \in\{\xi, \eta, \zeta\}$ we have $\left\langle\varepsilon_{1} g\right\rangle \leq 3\langle g\rangle$ and $\left\langle\varepsilon_{2} g\right\rangle \leq 7\langle g\rangle$. Combining these inequalities with the obvious analogue of the estimate (14) yields $\left\langle S_{(3)} \alpha\right\rangle \leq(3+3+9+$ $1)\langle\alpha\rangle=16\langle\alpha\rangle$. In a similar way, $\left\langle S_{(2)} \alpha\right\rangle \leq 11\langle\alpha\rangle$ and $\left\langle S_{(1)} \alpha\right\rangle \leq 4\langle\alpha\rangle$. This extends to all $X \in\left\{R^{ \pm 1}, S^{ \pm 1}, T^{ \pm 1}\right\}$, and hence $\kappa \leq 16$. Using these facts as ingredients in Theorem 6 we obtain:

Theorem 10. Let $(l, m, n)$ be a hyperbolic triple. Then, for every positive integer $\delta$ there exists an epimorphism $\varphi: T(l, m, n) \rightarrow H$ of injectivity radius at least $\delta$ such that $|H| \leq C^{\delta}$ where $C \leq 2^{32 l m n}$.

A further improvement on the exponent at $C$ can be obtained by observing that the above calculations remain valid if, for $k \in\{l, m, n\}$, the polynomial $\left(x^{2 k}-1\right) /\left(x^{2}-1\right)$ is replaced with $x^{k}+1$ or $\left(x^{k}+1\right) /(x+1)$, depending on whether $k$ is even or odd. This way the dimension of $A$ decreases by a factor of 8 , giving the bound $C \leq 2^{4 l m n}$.

The planar width version of the above result reads as follows.

Corollary 11. Let $(l, m, n)$ be a hyperbolic triple and let $\mu=\max \{l, m, n\}$. Then, for every positive integer $d$ there exists a regular hypermap of type $(l, m, n)$ of planar width larger than $d$, the number of vertices of which does not exceed $2 \tilde{C}^{d}$ where $\tilde{C} \leq 2^{2 \mu l m n}$.

We bypass the problem of comparing the bounds from Theorems 8 and 10 by establishing yet another bound that is asymptotically better. At this place we just note that a naive experience with cyclotomic polynomials might suggest the false impression that coefficients of the $k$-th cyclotomic polynomial are always small compared to its degree. However, in [16] it was proved that the natural logarithm of the norm of the $k$-th cyclotomic polynomial is larger than $\exp (\ln 2 \ln k / \ln \ln k)$ for infinitely many $k$. 


\section{Refining the calculations}

The bounds on planar width obtained so far were extracted from the fact that $\langle X \alpha\rangle \leq \kappa\langle\alpha\rangle$ implies $\left\langle X^{k} \alpha\right\rangle \leq \kappa^{k}\langle\alpha\rangle$. In this section we use a more direct approach. We begin with determining the powers of the matrix $S$.

Proposition 12. Let $A$ be a $\mathbb{Z}$-algebra, let $\zeta_{*}, \eta_{1}, \xi_{1} \in A$, and let $\zeta_{i}=\zeta_{*}^{i}+\zeta_{*}^{-i}$. Assume that $\zeta_{*}^{2}-1$ is invertible. Then, for the matrix $S$ from (3) and any positive integer $k$ we have

$$
S^{k}=\left(\begin{array}{ccc}
-1-\sum_{i=1}^{k-1} \xi_{2 i} & -\sum_{i=1}^{k} \xi_{2 i-1} & 0 \\
\sum_{i=1}^{k} \xi_{2 i-1} & 1+\sum_{i=1}^{k} \xi_{2 i} & 0 \\
\eta_{1} \Sigma_{1}+\zeta_{1} \Sigma_{4} & \eta_{1} \Sigma_{2}+\zeta_{1} \Sigma_{3} & 1
\end{array}\right)
$$

and

$$
S^{-k}=\left(\begin{array}{ccc}
1+\sum_{i=1}^{k} \xi_{2 i} & \sum_{i=1}^{k} \xi_{2 i-1} & 0 \\
-\sum_{i=1}^{k} \xi_{2 i-1} & -1-\sum_{i=1}^{k-1} \xi_{2 i} & 0 \\
\eta_{1} \Sigma_{3}+\zeta_{1} \Sigma_{2} & \eta_{1} \Sigma_{4}+\zeta_{1} \Sigma_{1} & 1
\end{array}\right)
$$

where $\Sigma_{1}=k+\sum_{i=1}^{k-1}(k-i) \xi_{2 i}, \Sigma_{2}=\sum_{i=1}^{k}(k+1-i) \xi_{2 i-1}, \Sigma_{3}=k+\sum_{i=1}^{k-1}(k-i) \xi_{2 i}$, and $\Sigma_{4}=\sum_{i=1}^{k-1}(k-i) \xi_{2 i-1}$.

Proof. Although it is possible to use induction, we sketch a more elegant way inspired by the spectral properties of $S$. As we saw in Section 3, $S$ is the matrix of an affine transformation given by $\bar{u} \mapsto \bar{u} L+\bar{a}$. Consequently, $S^{k}$ represents the mapping $\bar{u} \mapsto \bar{u} L^{k}+\bar{a}(I+L+$ $\left.\ldots+L^{k-1}\right)$. Realising that

$$
L=P^{-1} D P=\frac{1}{1-\zeta_{*}^{2}}\left(\begin{array}{cc}
1 & -\zeta_{*} \\
-\zeta_{*} & 1
\end{array}\right)\left(\begin{array}{cc}
\zeta_{*}^{2} & 0 \\
0 & \zeta_{*}^{-2}
\end{array}\right)\left(\begin{array}{cc}
1 & \zeta_{*} \\
\zeta_{*} & 1
\end{array}\right)
$$

we obtain

$$
L^{k}=P^{-1} D^{k} P=\frac{1}{1-\zeta_{*}^{2}}\left(\begin{array}{cc}
1 & -\zeta_{*} \\
-\zeta_{*} & 1
\end{array}\right)\left(\begin{array}{cc}
\zeta_{*}^{2 k} & 0 \\
0 & \zeta_{*}^{-2 k}
\end{array}\right)\left(\begin{array}{cc}
1 & \zeta_{*} \\
\zeta_{*} & 1
\end{array}\right)
$$

which, after evaluation of the product, leads to the expression for the linear part of $S$.

A fast way to deal with the affine part of $S$ is based on the following four relations involving eigenvectors:

$$
\begin{aligned}
\bar{a} & =\eta_{1}\left(1, \zeta_{1}\right)+\xi_{1}(1,0), \\
\left(1, \zeta_{1}\right) & =\left(1, \zeta_{*}\right)+\left(1, \zeta_{*}^{-1}\right)-(1,0), \\
\left(1, \zeta_{*}\right) L & =\zeta_{*}^{2}\left(1, \zeta_{*}\right), \text { and } \\
\left(1, \zeta_{*}^{-1}\right) L & =\zeta_{*}^{-2}\left(1, \zeta_{*}^{-1}\right) .
\end{aligned}
$$

Details of the computations are left to the reader.

In order to effectively utilise the above result in Representation 2, we have to improve the bound (11). We continue with a technical lemma, presented in a more general form than actually needed here. 
Lemma 13. Let $f(x) \in \mathbb{Z}[x]$ be a monic, reciprocal polynomial of even degree, dividing $x^{2 k}-1$. Let $\theta_{i}=x^{i}+x^{-i}+(f(x)) \in \mathbb{Z}[x] /(f(x))$. Then, for every $j$ such that $1 \leq j<k$ and every $a_{0}, a_{1}, \ldots, a_{k} \in \mathbb{Z}$,

$$
\begin{aligned}
& \theta_{j}\left(a_{0}+\sum_{i=1}^{k} a_{i} \theta_{i}\right)= \\
& \begin{array}{r}
a_{j}+a_{j-1} \theta_{1}+\ldots+a_{1} \theta_{j-1}+a_{0} \theta_{j}+a_{1} \theta_{j+1}+\ldots+a_{k-j-2} \theta_{k-2}+ \\
a_{k-j-1} \theta_{k-1}+a_{k-j} \theta_{k}+a_{j}+a_{j+1} \theta_{1}+\ldots+a_{k-1} \theta_{k-j-1}+a_{k} \theta_{k-j}+ \\
\quad a_{k-1} \theta_{k-j+1}+\ldots+a_{k-j+2} \theta_{k-2}+a_{k-j+1} \theta_{k-1}+a_{k} \theta_{k-j} .
\end{array}
\end{aligned}
$$

Proof. First, let us note that $f(x) \mid x^{2 k}-1$ implies that $x^{i}+(f(x))=x^{2 k-i}+(f(x))$, and consequently $\theta_{i}=\theta_{2 k-i}$. We evaluate the product on the left-hand side of the equation in the lemma by first applying (6) and then rearranging:

$$
\begin{gathered}
\theta_{j}\left(a_{0}+\sum_{i=1}^{k} a_{i} \theta_{i}\right)=a_{0} \theta_{j}+\sum_{i=1}^{k} a_{i} \theta_{j-i}+\sum_{i=1}^{k} a_{i} \theta_{j+i}= \\
{\left[a_{j}+\left(\sum_{i=1}^{j-1} a_{i} \theta_{j-i}\right)+a_{0} \theta_{j}+\left(\sum_{i=1}^{k-j} a_{i} \theta_{j+i}\right)\right]+\left[a_{j}+\left(\sum_{i=j+1}^{k} a_{i} \theta_{j-i}\right)+\left(\sum_{i=k-j+1}^{k-1} a_{i} \theta_{j+i}\right)+a_{k} \theta_{j+k}\right] .}
\end{gathered}
$$

The two lines of the formula in the lemma can now be easily obtained by applying the relations $\theta_{i}=\theta_{-i}=\theta_{2 k-i}$ to the two expressions that are enclosed in square brackets.

We now use Lemma 13 to the situation in Representation 2 with the $\mathbb{Z}$-algebra $A=$ $\mathbb{Z}[\xi, \eta, \zeta]$, that is, we will consider $\theta \in\{\xi, \eta, \zeta\}$ and $k \in\{l, m, n\}$. We will show details of the computations only for $\theta=\zeta, k=n$, and for elements of the form $g(\zeta)=a_{0}+\sum_{i=1}^{n-2} a_{i} \zeta_{i}$. Since in our basis for Representation 2 the elements $\zeta_{i}$ appear only with subscripts $i \leq n-2$, the term $a_{n} \zeta_{n-j}$ is not present at all in the formula of Lemma 13. Taking the norm of both sides of the formula and using (11) we obtain $\left\langle\zeta_{j} g\right\rangle \leq\left(2+2\left\langle\zeta_{n-1}\right\rangle+\left\langle\zeta_{n}\right\rangle\right)\langle g\rangle \leq$ $(2+2+4)\langle g\rangle=8\langle g\rangle$ for every $g \in A$ and $1 \leq k \leq n-1$.

Using the standard approach combined with the above results we obtain

$$
\left\langle S^{k} \alpha\right\rangle \leq\left(3\left[2 k+8\left(\frac{k(k+1)}{2}+3 \frac{k(k-1)}{2}\right)\right]+1\right)\langle\alpha\rangle=\left(48 k^{2}-18 k+1\right)\langle\alpha\rangle
$$

Recalling that because of the order of $S$ we are only interested in the values $k \leq n / 2$, we conclude that for any power $X$ of $S$ we have

$$
\frac{\langle X \alpha\rangle}{\langle\alpha\rangle} \leq 12 n^{2}-9 n+1 \leq 16 n^{2}
$$

Combining this with the arguments used in the proof of Theorem 6 we finally arrive at:

Theorem 14. Let $(l, m, n)$ be a hyperbolic triple and let $\mu=\max \{l, m, n\}$. Then, for every positive integer $d$ there exists a regular hypermap of type $(l, m, n)$ of planar width larger than $d$, the number of vertices of which does not exceed $2 \tilde{C}^{d}$ where $\tilde{C} \leq(4 \mu)^{64 l m n}$. 
This is in general much better than the bound from Corollary 11, since in the exponent the term $2 \mu$ has been replaced with $64\left(2+\log _{2} \mu\right)$.

Let us sum up the advantages and possible disadvantages of the two representations we have been using throughout. A strong point for Representation 1 is a smaller dimension of the algebra, especially when g.c.d. $(l, m, n)$ is relatively large compared to $l, m, n$. Moreover, Representation 1 enables one to work over the $\operatorname{ring} \mathbb{Z}[2 \cos (\pi / M)]$, which is a ring of algebraic integers. We have used the fact that this ring embeds in a field, and there are other important properties of such rings that could be useful in future research in this area. A weaker point of Representation 1 is the potentially large upper bound on $\kappa$. On the contrary, the corresponding bound on $\kappa$ in Representation 2 is small, even constant. This advantage is, however, negatively outweighed by large dimension of the algebra. The effect of this becomes significant if g.c.d. $(l, m, n)$ is relatively large compared to $l, m, n$. Also, the algebra is bound to have divisors of zero.

In what follows we will show that the drawback of potentially large exponents $\kappa$ in Representation 1 can be overcome. This will be achieved by applying Lemma 12 to Representation 1 with the goal to obtain bounds on $C$ comparable to the ones appearing in Representation 2.

First, we extend the definition of a norm to an arbitrary generating set $\left\{\beta_{i} ; i \in \mathcal{I}\right\}$ of a $\mathbb{Z}$-algebra $A$ by setting

$$
\langle g\rangle=\min \left\{\max _{i \in \mathcal{I}}\left|a_{i}\right| ; g=\sum_{i \in I} a_{i} \beta_{i}, a_{i} \in \mathbb{Z}\right\}
$$

where the minimum is taken over all possible ways of expressing $g$ as an integral linear combination of generators. It is easy to see that for any two norms \langle\rangle$_{1}$ and \langle\rangle$_{2}$ with respect to two finite generating sets of a $\mathbb{Z}$-algebra $A$ there exists a constant $c$ such that for every $g \in A$ we have

$$
\langle g\rangle_{1} \leq c\langle g\rangle_{2} .
$$

Let $(l, m, n)$ be a hyperbolic triple, with $M=1 \cdot \operatorname{com}(l, m, n)=l_{0} l=m_{0} m=n_{0} n$ and $\mu=\max \{l, m, n\}$. Consider the $2 M$-th cyclotomic polynomial $f(x) \in \mathbb{Z}[x]$. As in Representation 1 before, in the ring $\mathbb{Z}[x] /(f(x))$ we let $\theta=x+x^{-1}+(f(x))$ and $\theta_{i}=$ $x^{i}+x^{-i}+(f(x))$. Further, we define $\xi=\theta_{l_{0}}, \eta=\theta_{m_{0}}$, and $\zeta=\theta_{n_{0}}$. Our underlying $\mathbb{Z}$-algebra will be $A=\mathbb{Z}[\theta]$. Let $R, S$, and $T$ be matrices as in (3) where $\xi, \eta$, and $\zeta$ are as defined above. The new idea is to work with two norms simultaneously. The first norm \langle\rangle wlll be the previously used integral norm with respect to the basis $\left\{1, \theta_{1}, \theta_{2}, \ldots, \theta_{u-1}\right\}$ of $A$ where $u=\phi(2 M) / 2$. The second norm [ ] will be the integral norm with respect to the generating set $\left\{1, \theta_{1}, \theta_{2}, \ldots, \theta_{M}\right\}$.

Yet another application of the methods permeating this paper gives the following result the proof of which is left to the reader.

Lemma 15. Let $g(\theta) \in A$ and $X \in\left\{R^{ \pm 1}, S^{ \pm 1}, T^{ \pm 1}\right\}$. Then, for any integer $k$,

(1) $[g] \leq\langle g\rangle \leq(3+\langle f\rangle)^{M-u+1}[g]$,

(2) $\left[\theta_{i} g\right] \leq 3[g]$ for $1 \leq i<M$,

(3) $[X \alpha] \leq 16[\alpha]$,

(4) $\left[X^{k} \alpha\right] \leq(9 / 2) \mu^{2}[\alpha]$. 
These inequalities imply that for an arbitrary product $X^{\prime}=X_{1} X_{2} \ldots X_{\delta}$, where $X_{i} \in$ $\left\{R^{ \pm 1}, S^{ \pm 1}, T^{ \pm 1}\right\}$, we have $\left[X^{\prime}\right] \leq 16^{\delta-1}$ and $\left\langle X^{\prime}\right\rangle \leq(3+\langle f\rangle)^{M-u+1} 16^{\delta-1}$. Leaving the reiterating details aside, as the result we obtain the following conclusion.

Theorem 16. Let $(l, m, n)$ be a hyperbolic triple and let $M=$ l.c.m. $(l, m, n)$. Let $f(x)$ be the $2 M$-th cyclotomic polynomial. Then, for every positive integer $\delta$ there exists an epimorphism $\varphi: T(l, m, n) \rightarrow H$ of injectivity radius at least $\delta$ such that $|H| \leq D \cdot C^{\delta}$ where $D \leq(3+\langle f\rangle)^{4 M \phi(2 M)}$ and $C \leq 2^{16 \phi(2 M)}$.

The key point, however, on our way to further refinements is to consider more general exponents in the product in conjunction with Proposition 12. If $X^{\prime \prime}=X_{1}^{j_{1}} X_{2}^{j_{2}} \ldots X_{d}^{j_{d}}$, then $\left[X^{\prime \prime}\right] \leq\left(9 \mu^{2} / 2\right)^{d-1} \mu / 2$, and $\left\langle X^{\prime \prime}\right\rangle \leq(3+\langle f\rangle)^{M-u+1}\left(9 \mu^{2} / 2\right)^{d-1} \mu / 2$. Again, for every $\delta$ we may choose a prime $p$ such that $(3+\langle f\rangle)^{M-u+1}(4 \mu)^{2 d-1}+1<p<(3+$ $\langle f\rangle)^{M-u+1}(4 \mu)^{2 d}$, making the norm $\left\langle X^{\prime \prime}-I\right\rangle$ smaller than $p$. Invoking Theorem 6 and its proof we eventually obtain:

Theorem 17. Let $(l, m, n)$ be a hyperbolic triple, let $M=$ l.c.m. $(l, m, n)$, and let $\mu=$ $\max \{l, m, n\}$. Let $f(x)$ be the $2 M$-th cyclotomic polynomial and let $\langle f\rangle$ be the largest absolute value of its coefficients. Then, for every positive integer $d$ there exists a regular hypermap of type $(l, m, n)$ of planar width larger than $d$ with number of vertices not exceeding $2 D \cdot \tilde{C}^{d}$ where $D \leq(3+\langle f\rangle)^{2 M \phi(2 M)}$ and $\tilde{C} \leq(4 \mu)^{8 \phi(2 M)}$.

We note that even though the constant $D$ looks enormous, its effect with increasing $\delta$ becomes insignificant. Therefore the last two bounds are asymptotically better than the bounds of Theorem 10 and Theorem 14, respectively.

\section{Maps, Hurwitz groups, and conclusion}

We have seen in the Introduction that the research into injectivity radius of epimorphisms of triangle groups was actually motivated by questions about planar width of regular maps rather than hypermaps. Recall that a regular map of type $(m, n)$ can be identified with a regular hypermap of type $(2, m, n)$. If the triple $(2, m, n)$ is hyperbolic, that is, if $1 / m+1 / n<1 / 2$, the pair $(m, n)$ is said to be hyperbolic as well. The usual topological representation of a regular map is obtained from the face-3-coloured embedding of the corresponding hypermap by contracting the the faces coloured 2 to points and collapsing the quadrangles coloured 1 to line segments, giving rise to vertices and edges of the map, respectively. Accordingly, the group $T(2, m, n)$ is usually presented as a two-generator group in the form $T(2, m, n)=\left\langle r, s \mid r^{m}=s^{n}=(r s)^{2}=1\right\rangle$. This change of viewpoint affects the definition of injectivity radius and planar width. In particular, the injectivity radius of an epimorphism $\phi: T(2, m, n) \rightarrow H=\left\langle\rho, \sigma \mid \rho^{m}=\sigma^{n}=(\rho \sigma)^{2}=\ldots=1\right\rangle$ is defined with respect to the set $\left\{r, r^{-1}, s, s^{-1}\right\}$. The topological definition of planar width of regular maps carries over in the obvious way, that is, it is again the smallest number of intersections of a non-contractible closed curve with the 1-skeleton of the map. Since the 1-skeleton of the map is different from the 1-skeleton of the corresponding hypermap, the algebraic characterisation of planar width is slightly different as well and reads as follows. The planar width of the map associated with an epimorphism $\varphi: T(2, m, n) \rightarrow H$ is the smallest positive integer $d$ such that there exists $w=\left(r^{i_{1}} s^{j_{1}}\right)\left(r^{i_{2}} s^{j_{2}}\right) \ldots\left(r^{i_{d}} s^{j_{d}}\right)$ such that $w \neq 1$ and $\varphi(w)=1$.

Estimates on injectivity radius of epimorphisms of $T(2, m, n)$ and on planar width of regular maps can be obtained as consequences of our general approach. One may, of course, 
substitute the value $l=2$ in all our previous results. A better way, however, is to observe that the fact that $2 \cos (\pi / 2)=0$ means that in every representation of $T(2, m, n)$ based on the matrices $R, S$, and $T$ given in (3), we can use $\xi=0$ and, of course, ignore $T$. Carrying the preceding computation through with such $R$ and $S$ one obtains the following specific versions of our earlier results.

Theorem 18. Let $(m, n)$ be a hyperbolic pair and let $M=1 \cdot \operatorname{com}(m, n)$. Then, for any positive integer $\delta$ there exists a representation of $T(2, m, n)$ of injectivity radius at least $\delta$ into a finite group of order at most $D \cdot C^{\delta}$, where $C \leq 13^{4 \phi(2 M)}$ and $D$ depends only on $m$ and $n$, and not on $\delta$.

Theorem 19. Let $(m, n)$ be a hyperbolic pair and let $M=1 \cdot \operatorname{co}(m, n)$. Then, for any positive integer $d$ there exists a regular map of type $(m, n)$ of planar width larger than $d$ with automorphism group of order at most $D \cdot \tilde{C}^{\delta}$, where $\tilde{C} \leq(5 m n)^{8 \phi(2 M)}$ and D depends only on $m$ and $n$, and not on $d$.

The reader may have realised that there is still room for further improvement of the presented methods and results. Our intention, however, was to focus on the main ideas and not to get stuck in details. Nevertheless, we at least indicate an improvement for the hyperbolic pair $(3,7)$, which plays an important role in the theory of maps. The finite quotients of $T(2,3,7)$ are known as the Hurwitz groups.

From the last part of the proof of Theorem 6 we see that the estimate on the order of the target group in a representation of $T(2,3,7)$ depends on three parameters: The type of the groups in which the representation takes place, dimension of the algebra $A$, and the prime $p$. We now show a way to reduce all three parameters in the case of $T(2,3,7)$. First, since the representation is, in fact, in the orthogonal group, the number $8=3^{2}-1$ can be reduced to 3 . Second, realising that $2 \cos (\pi / 2)=0$ and $2 \cos (\pi / 3)=1$, we can work in the ring $\mathbb{Z}[2 \cos (\pi / 7)]$ instead of $\mathbb{Z}[2 \cos (\pi / 42)]$, decreasing thereby the dimension of the algebra from 12 to 3. Third, a detailed evaluation of the products $R^{i} S^{j}$ instead of using just the powers $R^{i}$ and $S^{j}$ enables us to show that for the injectivity radius at least a given positive integer $\delta \geq 2$ it suffices to choose a prime $p$ such that $2 \cdot 5^{\delta-1}+1<p<4 \cdot 5^{\delta-1}$. Similarly, for planar width at least $d \geq 1$ is is sufficient to choose $p$ satisfying $6 \cdot 21^{d-1}+1<p<12 \cdot 21^{d-1}$. Putting the pieces together gives the following results, leaving the technical details to the reader.

Theorem 20. For any positive integer $\delta \geq 2$ there exists a representation of $T(2,3,7)$ of injectivity radius at least $\delta$ into a finite group of order at most $5^{9 \delta}$

Theorem 21. For any positive integer $d$ there exists a regular map of type $(3,7)$ of planar width larger than $d$ with automorphism group of order at most $21^{9 d}$.

Even though the constants $5^{9}$ and $21^{9}$ are still rather large, they are considerably smaller than the constants $13^{48}$ and $105^{96}$, obtained by substituting the values $m=3$ and $n=7$ in the bounds of Theorems 18 and 19.

We conclude with a few remarks. It may be instructive to sum up the individual stages leading from the original upper bound in [14] through the intermediate steps in $[1,5]$ to the improvements we presented in the last three section. The innovation of $[1,5]$ was in considering representations over factor rings of multivariate polynomials and a more accurate 
evaluation of the bounds at the expense of compactness of the obtained formulae. The contribution of the present paper is threefold. First, we derived a rigorous algebraic background for the ideas used in the previous papers. Second, utilisation of variables of the form of a sum of an element and its inverse enabled us to abandon Tchebyshev's polynomials and, instead, work with polynomials $\left(x^{2 k}-1\right) /\left(x^{2}-1\right)$ of norm 1 . The associated change of basis opened the room to working with powers of the generating matrices and led to direct computation of planar width. Third, extending the concept of norm to generating sets enabled us to return to representations over complex numbers which, at the same time, have a smaller dimension.

Our improvements on the estimates on $C$ and $\tilde{C}$, although significant, are still (roughly) exponential in $l m n$, while the lower bound extracted from [11] is polynomial in $l m n$. To improve the lower bound one would have to use some knowledge about fundamental regions of normal, torsion-free, finite-index subgroups of triangle groups; such regions are, in general, far from disk-neighbourhoods considered in [11]. As regards the upper bound, additional refinements of our methods combined by a use of different bases may result in further progress. A possibly fruitful direction could be to incorporate the classification of 2-dimensional linear representations of triangle groups over finite fields presented in [13]. Working with linear representations over $u$-dimensional algebras, however, implies that one will not be able to get away with the exponent $u$ appearing in the bound on $C$, due to Minkowski's theorem on lattice points in convex subsets of multidimensional spaces (see e.g. [7]).

Acknowledgement. The authors acknowledge partial support from the VEGA Grants No. 1/3020/06, 1/3022/06, 1/0489/08, and APVV Research Grants 20-000704, 0040-06, 010407, and LPP-0203-06.

\section{References}

[1] M. Abas, Homomorphisms of triangle groups with large injectivity radius, Acta Math. Univ. Comenianae 72 (2003), no. 2, 253-259.

[2] A. Breda, R. Nedela and J. Širáň, Classification of regular maps of negative prime Euler characteristic, Trans. Amer. Math. Soc. 37 (2005), no. 10, 4175-4190.

[3] K. S. Brown, Buildings, Springer, 1989.

[4] J. E. Humphreys, Reflection groups and Coxeter groups, Cambridge Univ. Press, Cambridge, 1990.

[5] M. Ipolyiová, Algebraic constructions of regular maps, Ph.D. Dissertation, 2004.

[6] L. A. James and G. A. Jones, Regular orientable imbeddings of complete graphs, J. Combinat. Theory Ser. B 39 (1985), 353-367.

[7] G. A. Jones and M. Jones, Elementary Number Theory, Springer-Verlag London, Ltd., London, 1998.

[8] G. A. Jones and D. Singerman, Belyı̆ functions, hypermaps, and Galois groups, Bull. London Math. Soc. 28 (1996), 561-590.

[9] A. Mal'cev, On the faithful representation of infinite groups by matrices, Russian: Mat. Sbornik 8 (1940), no. 50, 405-422; English: Amer. Math. Soc. Transl. 45 (1965), no. 2, 1-18.

[10] J. Mennicke, Eine Bemerkung über Fuchssche Gruppen, Invent. Math. 2 (1967), 301-305.

[11] J. F. Moran, The growth rate and balance of homogeneous tilings in the hyperbolic plane, Discrete Math. 133 (1997), no. 1-3, 151-186. 
[12] R. Nedela and M. Škoviera, Regular maps on surfaces with large planar width, European J. Combin. 22 (2001), no. 2, 243-262.

[13] Ch.-H. Sah, Groups related to compact Riemann surfaces. Acta Math. 123 (1969), 13-42.

[14] J. Širáň, Triangle group representations and constructions of regular maps, Proc. London Math. Soc. 82 (2001), no. 3, 513-532.

[15] J. Tits, Groupes et géométries do Coxeter, unpublished manuscript, 1961.

[16] R. C. Vaughan, Bounds for the coefficients of cyclotomic polynomials, Michigan Math. J. 21 (1974), 289-295. 